
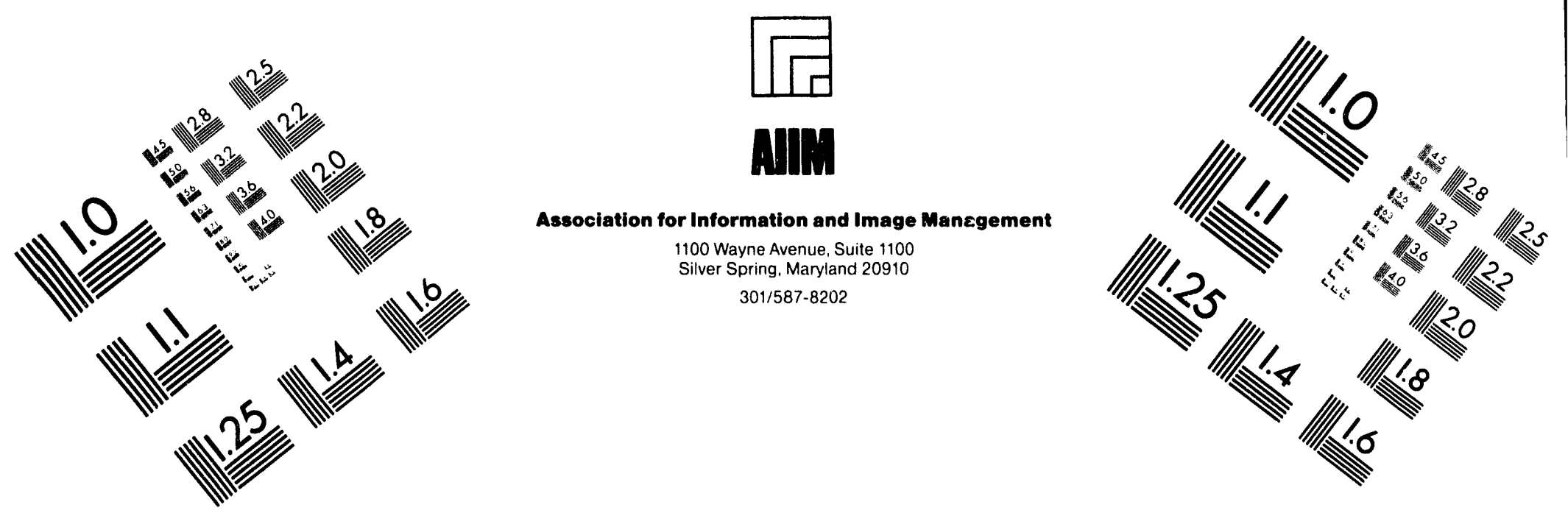

\title{
Centimeter
}

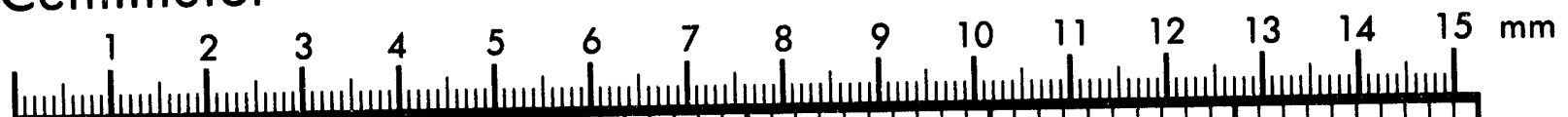

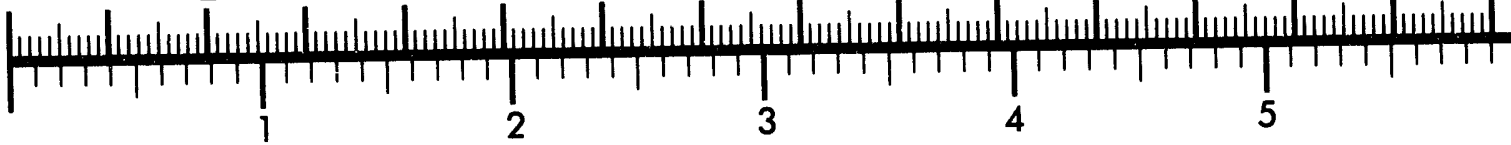
Inches
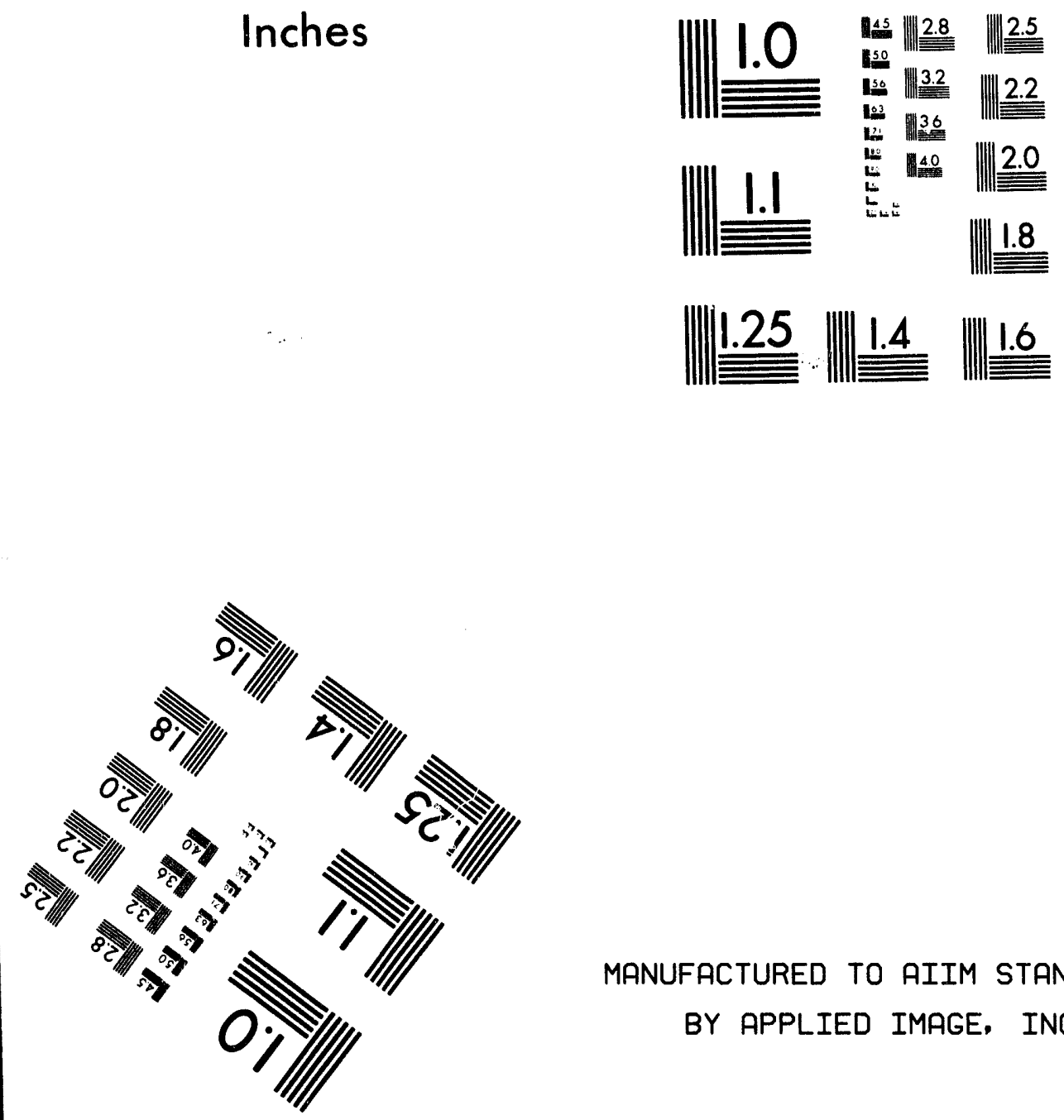

MANUFACTURED TO AIIM STANDARDS

BY APPLIED IMAGE, INC.

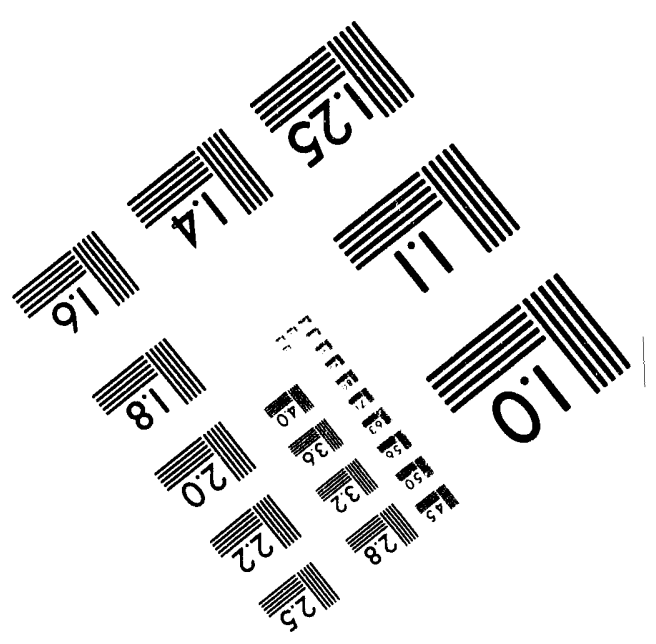



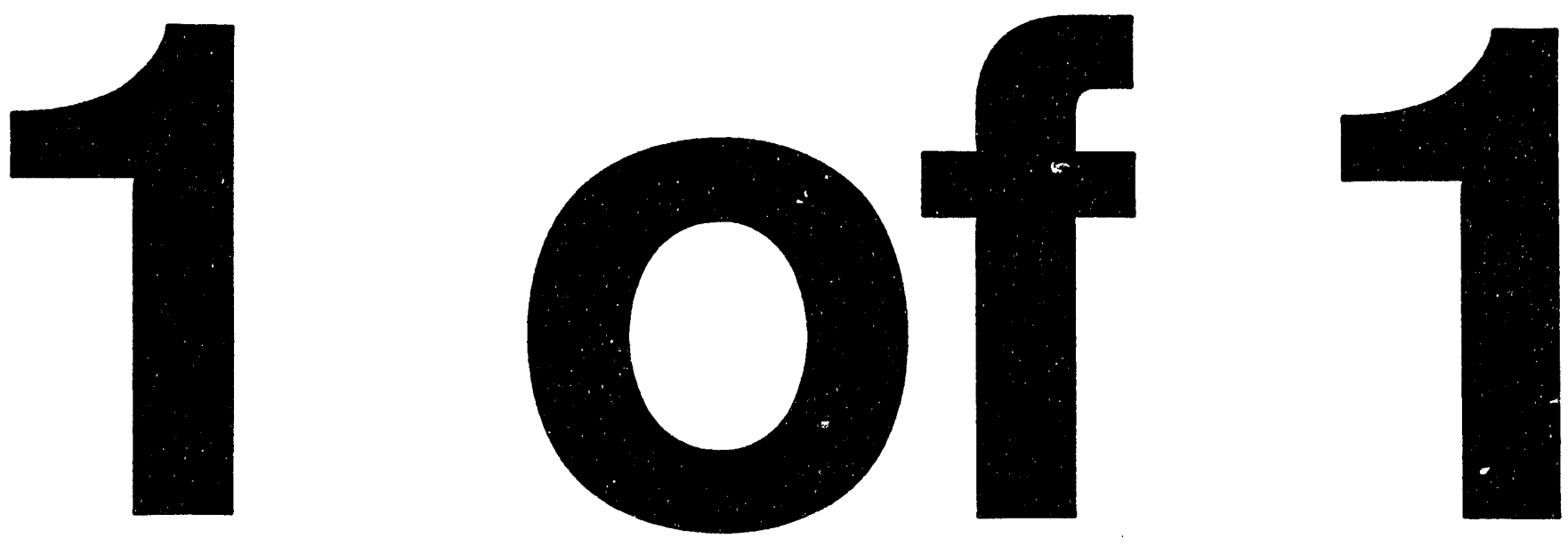
July 13, 1994

LBL-35880

\title{
The Integration of Mind into Physics *
}

\author{
Henry P. Stapp \\ Theoretical Physics Group \\ Lawrence Berkeley Laboratory \\ University of California \\ Berkeley, California 94720
}

\begin{abstract}
The proper goal of science is a unified theory of all of nature, including our thoughts. An adequate theory of this kind will resolve the quantum measurement problem, which is to reconcile the nonclassical character of the quantum world with the classical character of our perceptions of it. A framework for such a theory is described. It weds the opposing views of Bohr and Einstein. Bohr held that quantum theory provides rules that relate aspects of our knowledge, while Einstein claimed that basic theory should describe what could be reality itself, not merely our knowledge of it.
\end{abstract}

Presented at the conference:

Fundamental Problems in Quanturn Theory

Univ. of Maryland, June 18-22, 1994

Honoring Professor John Archibald Wheeler

"This work was supported by the Director, Office of Energy Research, Office of High Energy and Nuclear Physics, Division of High Energy Physics of the U.S. Department of Energy under Contract DE-AC03-76SF00098.

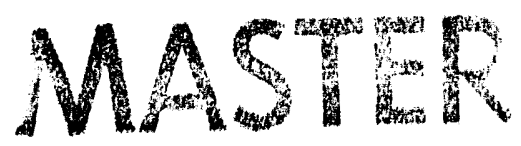




\section{A Theory of Mind and Matter}

John Wheeler has a knack for asking the right question. At the beginning of this conference he directed our attention to what he deemed to be a key question in the foundations of quantum theory:

$\mathrm{H} \phi$ ffding asked "Where can the photon be said to be?"

Bohr replied "To be. To be. What does it mean 'to be'?"

This paper proposes to answer this question in a way that yields a parsimonious theory of mind and matter that reconciles the opposing views of Bohr and Einstein. Bohr held that quantum theory describes relationships between aspects of our knowledge, while Einstein insisted that our basic theory describe what could be reality itself, not merely our knowledge of it.

Wheeler provided guidance in our search for an answer by offering several further quotations:

"The concept of the physical object is a convenient myth."

"Observations are the iron posts upon which everything is based; all else is papier mâché."

"No phenomenon is a phenomenon until it is an observed phenomenon."

Modern science grew out of Descartes' disjunction of mind and matter. But that separation led to classical physics, not to ultimate science. Mind is injected back into physics by quantum mechanics, for the basic problem in quantum mechanics is to reconcile the nonclassical character of the quantum world with the classical character of our perceptions of it.

Bohr confronted this problem by adopting an epistemological approach based on "our knowledge": he regarded the quantum formalism as merely a set of rules that give statistical predictions relating our classically describable perceptions. Yet Bohr's theory is limited in scope by its exclusion of biological systems. This exclusion entails that the physical carriers of our knowledge, namely our brains, are not represented within the quantum system described by the theory. This omission is the basic cause of the difficulties that beset Bohr's version of quantum theory. An adequate dynamical theory should contain representations of the things that need to be related, and this means, for basic theory, both the quantum micro-realities and the classically described experi- 
enced facts. Ideally, these two things should be represented within basic theory as two aspects of a seamless dynamical unity.

Von Neumann developed an alternative approach that extends quantum theory to the entire universe, including our brains. But he needed two dynamical processes:

Process I, consisting of abrupt "quantum jumps", called events; and

Process II, consisting of continuous deterministic evolution according to the Schroedinger equation.

As regards these jumps, von Neumann's main result was that each one could be placed at any one of a sequence of alternative locations along the chain of causal connections that lead from the micro world of elementary particles to the macroscopic level of brain activity that corresponds to our conscious thoughts: differences in the placement of the jump have virtually no effect on the predictions of quantum theory. But von Neumann noted that there is one placement of the jumps that is naturally singled out from all others, namely the placement at the level of brain functioning where our conscious thoughts enter. All other placements are ad hoc and artificial, and disrupt the natural linkage between the purely physical world and the quantum principles.

Wigner affirmed and reinforced the idea that the quantum jumps be placed exclusively at the level of brain action where conscious thoughts enter. This placement of the jumps reduces the triality consisting of the mental world, the classical world, and the quantum world to a duality composed of the mental world and the quantum world: the observer-independent classical level of description is eliminated, and hence the world of classically describable thoughts rides directly on the world of quantum potentialities. This brings von Neumann's theory into alignment with Bohr's, in the sense that in both theories the classically described perceived facts are linked to each other via a purely quantum mechanical system, without the introduction of an observer-independent classical level of being.

Wigner later recanted, claiming that the disruptive effects of the environment make quantum theory inapplicable to macroscopic systems. However, his argument is not conclusive. The effects of the environment on macroscopic systems have been studied in great detail in recent years. Interactions with the environment certainly produce a great loss of effective phase coherence, but the overall practical effect of these interactions is to convert the quantum state 
at the level of macro-variables to an approximate statistical mixture of states. Macro-variables are collective variables that carry a large mass compared to the energies of the disturbances coming from the environment. Thus interaction with the environment converts the quantum state, effectively and approximately, to a statistical mixture of a thin veneer of sluggish macro-variables riding on an ocean of tumultuous micro-variable activity. This conversion justifies in practice, in many cases, the use of classical statistical mechanics at the macro level, but it leaves unresolved the central problem: where do we place the jump that reduces the approximate statistical mixtures of classically describable macro-states to the individual state that we perceive?

There is no empirical evidence for the occurrence of jumps at any place other than the mind-brain interface. Hence there is no scientific basis for introducing other jumps. Certainly the goal of bringing our mental image of the macroworld into concordance with our notoriously fallible classical intuition is not a sufficient reason!

Jumps are definitely needed inside our brains ${ }^{4}$. Thus the law of parsimony, and the lack of a natural criterion that picks out any other location, enjoins us to place the quantum jumps exclusively at the mind-brain interface.

The question then arises: Which brains?

I "know" only that I myself am conscious. However, conversations with other human beings, and the writings of psychologists and philosophers, have convinced me that some other human beings are, in all probability, also conscious. In this connection it is important to recognize that the goal in science is not certainty: certainty is unattainable. We create general theories, test them, and use them if they work; we never verify them. Thus in view of the great similarities, both structurally' and behaviorally, of myself and other human beings it is reasonable to posit, as the foundation of a tentative theory of nature, that all normal and alert human beings have thoughts similar to my own.

According to Bohr "The task of science is to expand our experience and reduce it to order". Here the "our" is, in the first instance, the human race. Science is an on-going human endeavour, and the facts that it must coordinate are the facts defined by our collective experience. By taking only human brains to be associated with the emergence of the classically describable facts we obtain 
a theory that is maximally parsimonious with respect to human-based science: the facts that are defined within the theory are precisely the facts that need to be explained by the theory.

The theory specified in this way describes what could be reality itself, including our knowledge of it. Thus it meets Einstein's demand that basic theory describe a possible reality. The virtue of theories of this kind is that they must conform to the strong condition that they be able, in principle, to describe all of nature in a completely consistent way. This rules out pretenders, and retains theories that have a greater promise to carry us beyond what we presently know.

One can consider theories that differ from this 'standard' human-based one by having a larger set of brains that harbor quantum jumps: theories in which the set of human brains is augmented to include some nonhuman ones, such as dog brains or computer brains. In this connection I note the following:

1. Theories based on larger sets of brains lead to consequences that differ within the set of facts defined by human experience from those of the standard humanbased theory. This is because the additional brains produce additional quantum jumps, and these jumps, occurring outside human brains, will generally lead to eventual consequences also within human brains. This situation differs from the one in classical physics, where the occurrence or nonoccurrences of thoughts, per se, in other brains have no empirical consequences for me, because the laws of classical physics make no reference to subjective experiences. Thus, according to the ideas of classical physics, the occurrence or nonoccurrence of 'experiences' in conjunction with the activities of brain/body $B$ make no difference at all in the physical world, and hence no difference in the consciousness of anyone else. 2. Theories that allow jumps in nonhuman brains, although different in principle from the standard theory, are virtually identical to it in practice, within the realm of human experiences. This is a corollary of von Neumann's analysis. Thus for all practical scientific purposes we human beings can, without introducing any significant error, use the standard human-based theory, even if quantum jumps do actually occur in nonhuman brains: inclusion of those other jumps would make virtually no changes in the predictions pertaining to human experiences.

3. By virtue of point 2 any extension of the standard human-based theory to a theory with jump-possessing nonhuman brains has no secure scientific justif- 
cation. The standard human-based theory is the most parsimonious theory of nature consistent with my knowledge that I have thoughts, and that other human beings are structurally and behaviorally very similar to me, and therefore ought to be treated on a par with me in a general theory of nature.

4. "Science is a tiny island of knowledge in a vast ocean of nescience." Let us not pretend to know more than we do. There exists a huge collection of theories that are different from one another in principle but that, by presently available techniques, are indistinguishable in practice. In full awareness of this fact we can choose, tentatively, the one that is best adapted to the human scientific enterprise, namely the one that describes a possible reality in which the dynamically generated facts are exactly the facts specified by the experiences of the community of communicating human observers.

5. After we have developed a satisfactory detailed understanding of the connection between human brains and human thoughts we may be in a position to make a reasonable extrapolation to nonhuman brains. We may then wish to shift to what might seem by then to be a more reasonable theory.

6. Within this theory each train of thought is dynamically connected to the process going on contemporaneously in the associated brain, rather than, as in certain AI theories, to a computer program, which might be instantiated by a variety of alternative and different dynamical processes.

7. In contrast to behavioristic appruaches, the primary scientific data here are the facts specified by our collective experience.

8. Within this theory the history of the universe is defined only insofar as it is defined by the facts specified by accumulated human knowledge.

This formulation of quantum theory reduces the problem of quantum measurement to the problem of the dynamical connection of mind to brain. Twenty years ago such a "reduction" might have been tantamount to casting the problem out of science. Today that is not true. Scientific pursuit of the question of the relationship between brain process and conscious process has become an important confluence of interest among increasing numbers of brain scientists, psychologists, neuro-psychologists, philosophers of science, and quantum physicists. The topic is rightly a "hot" subject, because it bears directly on the core issue of our conception of ourselves: on the question of the relationship of our thoughts to our bodies and brains, and to the universe around us. 
An adequate quantum theory of the relationship between brain and mind must be:

1. Concordant with all results from brain science.

2. Concordant with all results from psychology.

3. Concordant with all results from neuro-psychology.

4. Concordant with the demand that the full structure of each thought (i.e., each consciously experienced event) be fully represented in the brain state actualized by the corresponding quantum event.

5. Concordant with the demand that the brain state associated with a thought have the functional character appropriate to that thought: e.g., my thought "I will now raise my arm" must actualize a brain state that, under normal conditions, will cause my arm to rise.

An outline of a theory of that appears to meet these requirements is given in ref. 4. In that book I followed Heisenberg and allowed the quantum jumps to occur also at the level of quantum measuring devices, such as Geiger counters. But this raises the question of the rule that fixes the exact placement of these external jumps. In the present work I have shifted to the position described above. But that shift makes no change in the theory of the mind-brain interaction given in ref. 4, which I now briefly summarize.

\section{Quantum Theory of Brain Events}

The motivation within psychology and brain science for this theory of brain events is described in ref. 4, and will not be repeated here. The essential postulate is that each human thought is an event that actualizes a particular pattern of activity in a human brain. This thought is said to belong to that brain, and it is represented in the physicists' description of nature by the action of a projection operator that projects the prior (Heisenberg picture) state vector onto its successor. This successor contains the pattern of brain activity that is actualized by the thought, and it contains none of the alternative possible thought-related patterns that according to the quantum analog of Newton's laws of motion could have occurred, but in fact did not occur. The pattern of brain activity actualized by the thought is called the brain correlate of the thought, and it must contain within its structure all of the information and structure that is present in the felt content of the thought.

Each rudimentary thought always represents an image of the self in its en- 
vironment, and it either updates or adds to the latest image, in the case of a thought that "attends", or it creates a projected (into the future) image of the self in its environment in the case of a thought that "intends". The latter sort of image forms a template for subsequent motor action. Each pattern of brain activity that is actualized by such an event persists for certain time, and is thereby "facilitated": it is strengthened in such a way that subsequent excitations of portions of this pattern tend to excite the whole, leading to associative recall. Most such patterns are largely prefabricated, in the sense that they are formed from earlier patterns, or their parts, joined together in new configurations. By virtue of the architecture of the brain, in conjunction with learning, the only allowed configurations are those that correspond to an image of a physically possible self in a physically possible environment, or to some generalization of this basic form. Details can be found in reference 4.

But what is the physical structure of the patterns of brain activity that are astualized by thoughts? In the first place, each one must be an enduring, and presumably oscillatory, pattern of activity that, through its composition in terms of subpatterns, represents all of the felt structure of the associated thought. Hence it must evidently cover a macroscopic portion of the brain. These spatially separated parts of the pattern are bound together dynamically: the entire structure hangs together as a resonating system by virtue of the mechanical feed-back and feed-forward linkages in the brain. As a nonlinear (at the classical level) system with an energy supply and feedbacks the system is non-stable in the sense that, like a system of microphone and amplifier, once the energy in the system passes a certain critical value it evolves rapidly into an oscillatory mode that soaks up all of the available power. Fatigue properties of neurons eventually cause the pattern to fade out, and hence the conscious brain advances, step-by-step, from one of these resonance states to the next.

No human being can predict the exact progression of these states. Even in a classical idealization such predictions are rendered impossible by our lack of knowledge of the unknown and uncontrollable effects of thermal noise and interactions with the environment. Hence our knowledge about the "next" state can be represented only by a probability function, even thou sh, according to classical ideas, this "next" state is completely fixed and predetermined. In a quantum world this lack of knowledge about the "next" state is elevated, 
through Heisenberg's principle of indeterminacy, to a matter of principle, and hence the form of the next state remains undetermined and indeterminate even in principle, until it is actualized by a thought.

\section{A Simplified Model of the Mind/Brain}

In order to make the ideas outlined above more concrete I shall describe a simplified model of the mind/brain. What I shall give is very much a toy model. It should be understood as a simplification of what was described in more detail in reference 4. (Ch.6 and Appendix.) Nevertheless, it may be useful, by starting at a still simpler stage, to bring out certain rudimentary features.

The "brain" will be taken to consist of: 1), a source of power, consisting, in this idealization, of a very massive simple harmonic oscillator; and 2), a set of simple harmonic oscillators that represent the different patterns of brain activity that are the alternative possibilities for what the next thought can actualize. Thus the classical unperturbed Hamiltonian is ${ }^{5}$

$$
H_{0}=\left(p^{2}+M^{2} \omega^{2} x^{2}\right) / 2 M+\sum_{i=1}^{n}\left(p_{i}^{2}+m^{2} \omega^{2} x_{i}^{2}\right) / 2 m .
$$

Note that I have taken the frequency of the power source, $\omega$, to be the same as the frequency of the modes $i$ that are the brain correlates of the possible thoughts. Introducing ${ }^{5}$

$$
a_{i}=p_{i} /(2 m \omega)^{1 / 2}-i(m \omega / 2)^{1 / 2} x_{i}
$$

and its complex conjugate $a_{i}^{*}$, and the analogous $a_{0}$ and $a_{0}^{*}$, one may re-write $H_{0}$ in the simpler form

$$
H_{0}=\sum_{i=0}^{n} \omega a_{i}^{*} a_{i}
$$

The interaction Hamiltonian has the form

$$
H_{1}=i \sum_{i=1}^{n}\left(a_{i}^{*} a_{0}-a_{0}^{*} a_{i}\right)\left(f_{i}-g_{i}\right),
$$

where $f_{i}$ and $g_{i}$ are positive real functions of the variables of the problem. Thus the Poisson bracket (i.e., classical) equation of motion ${ }^{6}$

$$
d u / d t=\{u, H\} \equiv-i \sum_{i}\left(\partial u / \partial a_{i} \partial H / \partial a_{i}^{*}-\partial H / \partial a_{i} \partial u / \partial a_{i}^{*}\right) \equiv-i[u, H]
$$


where $\left[a_{i}, a_{i}^{*}\right]=1$ etc., gives

$$
d a_{i} / d t=-i \omega a_{i}+a_{0}\left(f_{i}-g_{i}\right)+\left(a_{i}^{*} a_{0}-a_{0}^{*} a_{i}\right)\left[a_{i},\left(f_{i}-g_{i}\right)\right],
$$

which is also the Heisenberg (i.e., quantum) equation of motion if the final bracket is interpreted as the commutator $a_{i}\left(f_{i}-g_{i}\right)-\left(f_{i}-g_{i}\right) a_{i}$. The source/sink mode is supposed to carry a very large amount of energy. Thus we assume that $a_{0}=A \exp -i \omega t$, with $A$ very large, positive, and essentially constani. Then the ansatz $a_{i}=A_{i} \exp -i \omega t$, with $A_{i}$ real and positive, and the definitions $f_{i}=f_{i}^{\prime} / A$ and $g_{i}=g_{i}^{\prime} / A$, convert this equation of motion to

$$
d A_{i} / d t=f_{i}^{\prime}-g_{i}^{\prime}
$$

The term proportional to $f_{i}^{\prime}$ feeds energy from the power source into the mode $i$, whereas the term proportional to $g_{i}^{\prime}$ provides for dissipation: it gives the flow of energy back into the source (and sink) mode described by the variable $a_{0}$.

This power-supply term is required to have two main features. The first is that the coupling is to be nonlinear, and lead to a very rapid build up of the energy in a mode $i$ if a certain critical value of that energy is reached. The energy will build up to a point where an equilibrium with dissipation is reached. The second feature is that the coupling should tend to divert, eventually, virtually all of the power flowing into this set of modes $i$ into a single one of them. The rationale behind this second property is that the purpose of conscious thinking is to construct, as soon as possible, some single coordinated plan of action, and to initiate it. Thus at the classical level the conscious brain process should produce one single plan, not several conflicting plans. Hence the coupling should be such that it will lead fairly quickly, at the classical level, to a steady state where all of the available power is passing through just one of the oscillator modes $i$. At the quantum level, the upcoming thought belonging to this brain will be an event (i.e., a quantum jump) that actualizes such a state. In this state one, and only one, of these modes $i$ will be excited, and hence there will be a clear distinction, at the level of the brain, between the various alternative possibilities between which the conscious thought will decide. Moreover, the selected mode will have the energy to initiate the chosen plan of action. 
Several points are worth emphasizing right away. The first is that the oscillator coordinates $x_{i}$ are collective coordinates: each one represents the amplitude of an entire organized patterns of activity, not just the position of an individual particle, nor even an individual neuron pulse. Each variable $x_{i}$ can be likened to the angle of rotation of a wheel, or the displacement of the center of mass of a large pendulum. It is this whole pattern of activity that is the brain correlate of a possible thought, i.e., the brain activity that can at the quantum level be actualized by the next thought.

In the framework provided by classical physics it is hard to understand how such an extended pattern of activity could be one single thought. For the basic idea in classical physics is to reduce things at the fundamental level to tiny localized objects, or tu localized values of fields: each extended thing is regarded as fundamentally an aggregate of tiny fundamental localized parts. But a thought is, psychologically, one single unified entity. In James's words: "Your acquaintance with reality grows literally by buds or drops of perception. Intellectually and upon reflection you can divide these into components, but as immediately given, they come totally or not at all." ${ }^{77}$ The point here is that a thought is, at the level of its ultimate essence, exactly what is given, namely one single entity. But it is represented in terms of the motions of billions of particles that are scattered all over the brain. In classical physics an aggregation of localized interacting particles can certainly act as whole, but it is nevertheless conceived to be an aggregation of localized parts: at the level of its ultimate essence, it is an aggregation.

In quantum thinking this "wholeness", or binding, problem vanishes: the quantum event is one single thing, which, however, can actualize an extended pattern of brain activity. Indeed, in quantum theory a quantum event is allowed to actualize only a pattern that is sufficiently "macroscopic": otherwise the successful predictions of the theory will be lost.

Note also that the quantum thought can be regarded as playing an essentially creative role: by choosing to actualize together patterns that have not previously occurred together, the thought creates, via the process of facilitation, a newly composed pattern of brain activity that is a new thought, or idea. In fact, every thought is in this sense a new invention, created by that thought self. Of course, some thoughts are more radically inventive than others. 
Possible forms for $f_{i}$ and $g_{i}$ are

$$
=(C / A)\left(a_{i}^{*} a_{i}\right)^{2} / \sum_{j}\left(a_{j}^{*} a_{j}\right)^{2}
$$

and

$$
g_{i}=(D / A) a_{i}^{*} a_{i},
$$

with $C$ and $D$ positive constants satisfying $C \gg D$. Then the equation of motion reduces to

$$
d A_{i} / d t=C A_{i}^{4} / \sum_{j} A_{j}^{4}-D A_{i}^{2} .
$$

Let the largest of the $A_{i}$ be $A_{1}>1$. If $A_{1}$ is not too large then it will grow. Some other $A_{i}$ 's may also grow, but they cannot overtake $A_{1}$. When $A_{1}$ gete close to its upper limit at

$$
C A_{1}^{4} / \sum_{j} A_{j}^{4}=D A_{1}^{2}
$$

all of the other $A_{i}$ with $A_{i}>1$ will be decreasing. Hence $A_{1}$ will tend to its upper limit, whereas all of the other $A_{i}$ 's will tend to the neighborhood of zero.

The emergence of this particular mode was, in this classical description, a consequence of the particular initial conditions: a different initial condition would have led, in general, to a different final state. Due to the initial uncertainties, coming from our lack of knowledge, arising perhaps from thermal fluctuations, our knowledge about the final state will be represented by a probability function, even though, in the classical framework, the actual final state will be fixed and definite.

In the quantum generalization of this classical model the quantum indeterminacy will lead to a quantum state that is roughly a superposition of states $\psi_{i}$, one for each of the alternative possible final states $i$ occurring in the classical description. The state $\psi_{i}$ has one of the modes, mode $i$, highly excited, and all the others unexcited. These different quantum states correspond to distinct and well separated possibilities for the brain state, and also to different classically describable perceptions of the self-in-environment. A quantum jump will then occur: it will actualize one of these possibilities and eradicate all the others. 


\section{Reasons For Believing That The Mind-Brain Connection Is Quan-}

tum Mechanical

The association of our thoughts with quantum jumps was postulated, above, in order to resolve the quantum measurement problem; i.e., to provide a coherent conception of nature that parsimoniously accomodates the quantum character of the micro-world. But there is a long causal chain from the microscopic world of elementary particles to the macroscopic patterns of activity that correspond to our thoughts, and other ways of forming a coherent conception of nature might be entertained. However, there are at least three indications that this linking of the mind-brain problem to quantum theory is correct:

1. The parallel dual structures of mind/matter and the quantum world.

2. The occurrence of consciousness where choices are needed.

3. The unity of thoughts, in contrast to the local reductionistic character of classical physics.

As regards duality, it should be noted, first of all, that the quantum theory of the mind/brain is in complete harmony with the mind/brain identity theory. Thoughts are not only represented as aspects of the physicists' description of brain activity, they can reasonably be imagined to actually be aspects of brain activity. Indeed, with the classical image of the nature of physical systems now banished, we are invited to form a new image of what brains are actually made of. Part of what they are made of would seem to be a sort of objective tendency for a thought to occur. But the other part of what a brain is made of could be the actually occurring thoughts themselves. Indeed, how else can we make an aspect of brain activity 'actual' other than by identifying it with something that is truly real, and thoughts (including feelings etc.) are the only sorts of things that we know to be real. In a naturalistic scientific approach one will want a thought to be an actual constituent of the physical system described by the physical theory, i.e., by quantum theory, not some mysterious disconnected thing that hovers around outside the physical system described by the physical theory.

In the literature supporting the mind-brain identity theory the usual position seems be that mind-brain identity entails a monistic rather than dualistic 
ontology. This bias evidently stems from the notion that the correct physical theory is classical physics. But whereas the physical world of classical physics is monistic the physical world as described by quantum theory is basically dualistic. It has two kinds of entities, operators and states, that evolve according to two different laws of motion (in the Heisenberg picture), one continuous and deterministic, the other abrupt and stochastic. And it has two types of beingness: that of potentiality represented by the wave function, and that of the factuality fixed by the events. This dual character matches that of matter and thought: each mind/body has its own private subjective thoughts, and also a tendency to produce images of its public or objective aspects in the thoughts associated with other mind/bodies. That is, each mind/body has two different aspects; the private/subjective/mental aspect, and the public/objective/material aspect. This duality is not destroyed by admitting the identity of thoughts with certain dynamical components of the mind/brain system, if that dynamical system itself, by virtue of its quantum nature, is essentially dualistic.

As regards choice, it is a fact that thoughts occur where choices are apparently needed. But in classical physics there are no choices: everything is fixed at the birth of the universe. Hence thoughts play no role in the unfolding of nature; they are superfluous. In the quantum theory of the mind/brain our thoughts do occur in conjunction with choices between bona fide alternatives.

The issue of unity was discussed above.

\section{Summary and Conclusions}

Good science introduces no superfluous entities. Bohr followed this dictum: recognizing that the basic problem in the interpretation of quantum theory was the incompatibility of the formal quantum principles with the classical character of our experiences Bohr made our classically describable experiences, and the knowledge derived from them, the basis of his interpretation. He interpreted the quantum formalism as a procedure acting on this knowledge, and thereby avoided the need to draw any line in the external world between its quantum and classical parts.

The scope of Bohr's version of quantum theory was, however, limited by its exclusion of biological systems. Universalization of the quantum principles brings human beings and their brains into the quantum mechanically described 
system, and this converts the problem of the interpretation of quantum thec $y$ to the problem of the dynamical connection of conscious process to brain process. As in Bohr's approach, there is no need to introduce into the external world any classicalization process, or any exact classical variables. Such things are alien to the quantum principles, and are superfluous: they have no empirical ramifications, or if they do have such ramifications then these represent deviations from the "pure" quantum predictions. Of course, all macroscopic variables have, due to their interactions with the microscopic degrees of freedom of their environment, a strong tendency to become approximately classical in various ways, which can be specified, and this allows us to imagine that various macroscopic variables in the universe have reasonably well defined values even if they are not being observed by anyone, and have never been observed by anyone. However, this approximate effective-classicalization of macro-variables is an automatic consequence of quantum theory, and there is no need within science to make it exact, merely to satisfy our classical intuition. On the other hand, we do need to introduce quantum jumps at the the level of of our thoughts, for it is the occurrence of these thoughts that are, empirically, what the quantum probabilities are the probabilities of.

This formulation of quantum theory is a composite of elements coming from Bohr, Einstein, von Neumann, Wigner, Heisenberg, and William James. In particular it integrates:

1. Bohr's recognition that physical science rests on the empirical foundation of human thoughts that have classically describable content.

2. Einstein's demand that, to ensure sufficient scope, logical coherence, and an adequate foundation for future developments, our basic physical theory should describe something that at least could be reality itself.

3. Von Neumann's demonstration that the quantum jumps can be placed exclusive at that level of brain activity where our thoughts enter.

4. Wigner's interactionist view that the quantum jumps do occur at the brainmind interface, and that mind and matter interact there.

5. Heisenberg's linkage of quantum process to the idea that nature's process proceeds by deterministic continuous evolution of potentialities punctuated by abrupt actual events, where each actual event constitutes a choice between the various possibilities generated by the prior deterministic evolution. 
6. James's emphasis on the wholeness of our thoughts, and their close association with our choices.

The cited parts of the ideas of the above-named physicists differ in important ways from the full ideas of these scientists:

1. Bohr's own formulation of the idea that our classically describable knowledge is the foundation of physical theory was expressed in ways that emphasized the intersubjective agreement of the classically described aspects of our experience. Hence it provided an intimation, though no explicit claim, that there may be classically describable properties existing outside of our thoughts, even though no such things are brought into Bohr's formulation of quantum theory.

2. Einstein's view that basic physical theory should describe "reality" referred, in fact, to a reality that did not include our thoughts.

3. Von Neumann's main point was the practical equivalence of various possible placements of the quantum/classical divide. He did mention the special role of consciousness, but the significance of this remark was obscure. It was his close friend and colleague Wigner ${ }^{2}$ who put the clear "mind-matter interaction" interpretation on von Neumann's words.

4. Wigner initially espoused this "interactionist" view, but later 8 argued that quantum theory did not apply to the macroscopic systems (hence to brains) because of the large effects of noise. However, the effects of noise on macroscopic variables is primarily to effectively decompose the quantum state into an approximate statistical mixture of states with narrow wave packets in phase space. There is no reason to claim that the quantum principles, viewed as the rules that govern our model of reality, must break down at the points where, due to decoherence effects, their empirical verification becomes difficult in practice.

5. Heisenberg, though he used the concepts of potentia and actual events to describe reality, did not institute the tight connection proposed here between these "real" things and the quantum formalism: he continued to view the latter, in accordance with the Copenhagen interpretation, as a tool for making predictions about our observations.

Thus the approach to quantum theory being proposed here is not an amalgamation of the complete views of the above-named scientists.

I acknowledge very useful correspondence with A. Jadczyk. 


\section{References.}

1. Descartes, René. Discourse on Method and The Meditations. trans. F.E. Sutcliffe, Harmondsworth, 1974.

2. E. Wigner, E. 1962. In The Scientist Speculates. I.J. Good, Ed. Heinemann. London.

3. Von Neumann, J. 1955. Mathematical Foundations of Quantum Theory. Princeton University Press. Princeton, NJ.

4. Stapp, H.P. 1993. Mind, Matter, and Quantum Mechanics. Spinger-Verlag. Heidelberg; New York, NY.

5. Dirac, P.A.M. 1947. The Principles of Quantum Mechanics. 3rd edit. Clarendon Press. Oxford: Ch VI.

6. Dirac, P.A.M. ibid: Ch IV.

7. James, W. 1987. In William James, Writings 1902-1910. The Library of America, New York, NY.: 1061.

8. Wigner, E. 1983. Review of the Quantum Mechanical Measurement Problem. In Quantum Optics, Experimental Gravity, and Measurement Theory, Eds. P.Meystre and M.O. Scully, NATO ASI series, Physics, Series B 94: 58. 

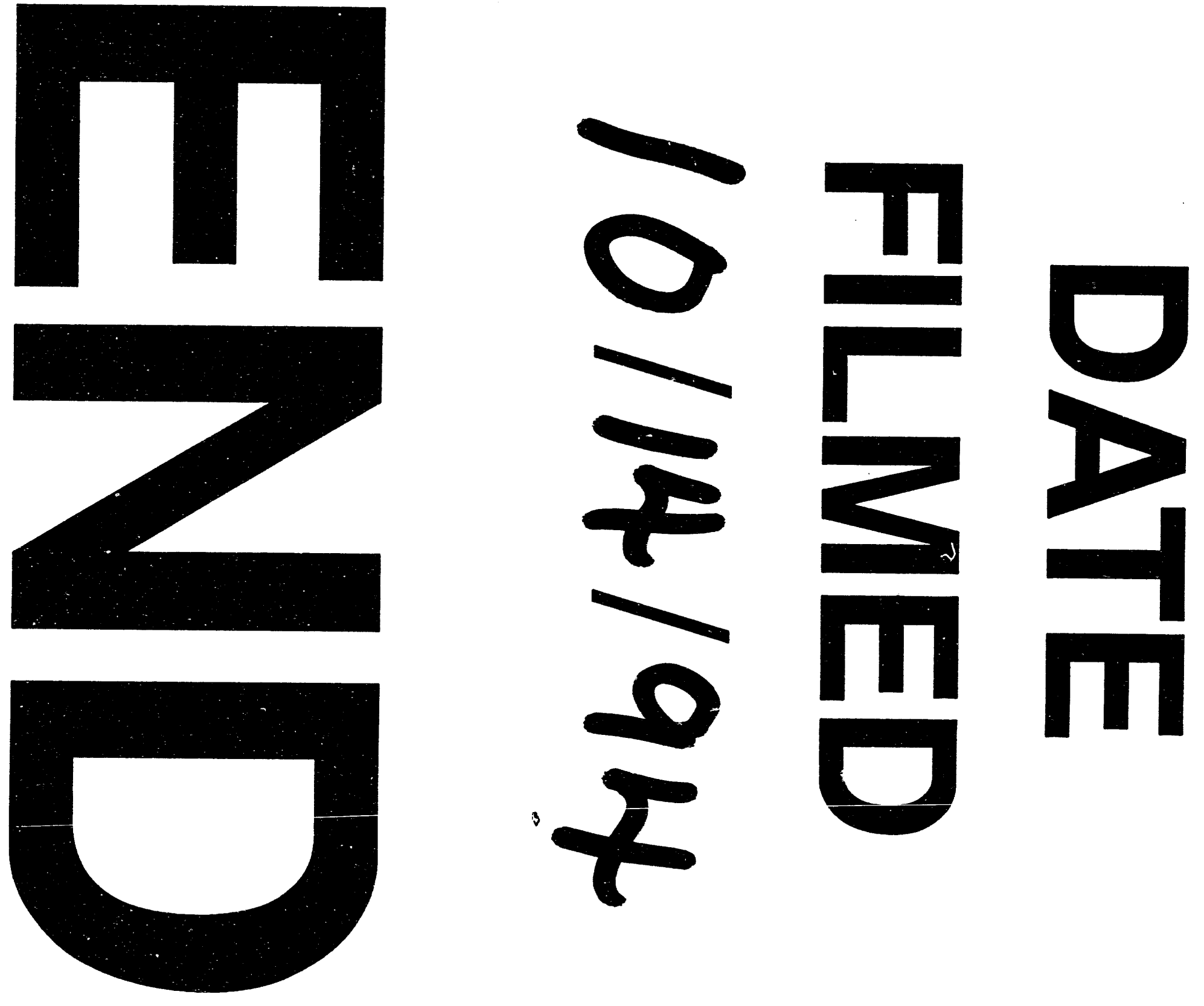
